\title{
On the Low Frequency Pressure Fluctuation in a 3/4 Open Jet Automotive Wind Tunnel
}

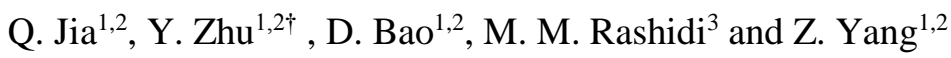 \\ ${ }^{1}$ Shanghai Automotive Wind Tunnel Center, Tongji University, Shanghai, 201804, China \\ ${ }^{2}$ Shanghai Key Lab of Vehicle Aerodynamics and Vehicle Thermal Management Systems, Shanghai, 201804, \\ China \\ ${ }^{3}$ Department of Civil Engineering, School of Engineering, University of Birmingham, Edgbaston, \\ Birmingham, B15 2TT, United Kingdom
}

†Corresponding Author Email: zyyllk0727@gmail.com

(Received August 13, 2018; accepted January 1, 2019)

\begin{abstract}
In the present research, a possible generation mechanism of low-frequency buffeting phenomenon based on a 1:15 open jet automotive wind tunnel was investigated. Evolution of vortex structures and pressure field in the plenum chamber have been visualized and analyzed by Large-eddy simulation (LES). It is shown that the low frequency pressure fluctuation is caused by the largescale structures and their interaction. Multiple proper orthogonal decomposition was adopted to analyze the flow field in the plenum chamber. The characteristic frequencies of the vortex-rings after pairing is the same as the dominant resonance of buffeting at this wind velocity.
\end{abstract}

Keywords: Wind tunnel; Buffeting; LES; PIV; POD.

\section{NOMENCLATURE}

$C C D \quad$ Charge-coupled Device

$C F D \quad$ Computational Fluid Dynamics

$C F L \quad$ Courant number

$D h \quad$ hydraulic diameter

$f \quad$ frequency

FFT Fast Fourier Transform

FOV Field of View

LES Large Eddy Simulation

$\begin{array}{ll}K-H & \text { Kelvin-Helmoltz } \\ P I V & \text { Particle Image Velocimetry } \\ P O D & \text { Proper Orthogonal Decomposition } \\ R e & \text { Reynolds number } \\ S t & \text { Strouhal number } \\ T & \text { cycle sequence time } \\ t & \text { transient time }\end{array}$

buffeting. For full-size automotive aeroacoustics wind tunnels, the main frequency of low frequency buffeting is often less than $10 \mathrm{~Hz}$, and the amplitude of pressure pulsation tends to exceed $100 \mathrm{~dB}$ (Amandolese et al., 2010). Accompanying the intense pressure pulsation is the velocity pulsation near the wind speed of $3 \%$ in the potential flow area. Although the frequency of these pressure pulsations are less than $20 \mathrm{~Hz}$ and cannot perceived by the human, they can affect acoustic measurements and psychoacoustic judgments. Meanwhile, velocity pulsations also affect the measurement of steady and unsteady aerodynamic characteristics. Severe pressure pulsations can even create dangerous additional loads and lead to damage to the wind tunnel structure. 
Therefore, research on the mechanism of low frequency buffeting has become a key to solve or control the low frequency buffeting phenomenon. However, current researches on low frequency buffeting mechanism is still in a blank state. It is generally recognized that the low-frequency buffeting is caused by the large-scale vortex structures in the jet shear layer. One mechanism is that high amplitude pressure pulsations occur when the preferred shedding frequency is the same as or close to the wind tunnel loop and the acoustic resonance mode of the plenum chamber. Another mechanism is that high-level pressure pulsations occur when the mode of a feedback cycle is the same or close to the acoustic resonance mode inherent in the wind tunnel. In this feedback loop, vortices interacts with the collector to generate a pressure wave backflow that stimulates the shear vortex shedding of the upstream jet, ie, the mode predicted by the Rossiter (1964) formula This mechanism is also similar to the mechanism of cavity resonance and wind noise in automotive windows.

Similar to the Karman vortex street, unstable waves can be observed in any free jet ejected from the nozzle. Near the outlet of the nozzle, unstable waves are dominated by high frequency and small scale vortices. When moving downstream, energy shifts from small-scale eddies to large-scale eddies, which in turn shifts the dominant frequency from high to low frequencies. This flow instability manifests itself in the jet as an axisymmetric vortex ring in the jet shear layer (Wickern et al., 2000). In 1985 , based on the open-jet wind tunnel of the German-Dutch Wind Tunnel (DNW) (Michel et al., 1985) measured a center distance between the central velocity of the nozzle and the hydraulic diameter of the nozzle in the downstream region of the open wind tunnel. $\mathrm{St} \approx 0.34$.

Rennie et al. (2000) modified the wind tunnel of the National Aeronautics Research Institute in Ottawa, Canada, before constructing a full-size automobile aeroacoustics wind tunnel of Hyundai Motor Company. The ratio of this model wind tunnel is $1 / 7$. The main purpose is to test a variety of means to suppress low-frequency buffeting, and also analyze the possible mechanism of pressure pulsation (Manuel et al., 1992; Arnette et al., 1999). The result given by the different jet lengths shows that the low-frequency buffeting phenomenon is related to the distance between the nozzle and the collector. Therefore, the feedback mechanism of the edge noise still plays a role in the low-frequency buffeting phenomenon. At the same time, the use of vortex shedding frequency $\mathrm{St}=0.34$ is not very accurate for prediction of low-frequency buffeting at different jet lengths. Therefore, afterwards he summarized a predictive formula that is similar to the model proposed by Morel et al. (1979) for the jet-hole model and the formula proposed by Rossiter et al. (1964) for the cavity model. Through this formula, Rennie and others have found that it is possible to more accurately predict the occurrence of low frequency buffeting at different jet lengths.

Amandolese et al. (2010) attributed this instability of the shear layer to the downstream pressure wave backflow and stimulated new upstream disturbances. While, some researchers didn't agree that the pressure wave is caused by the collision of the jet shear layer with the collecting port, due to the shape of the leading edge of the collector is a curved surface. The author's guess is that the collector needs to guarantee correct volumetric flux in order to satisfy the continuity condition. This could result in the pressure pulsations.

According to the above mentioned hypothetical mechanisms, the shedding of large-scale vortices in the jet shear layer and its interaction with the collector play an important role in studying the mechanisms of buffeting. In fact, the use of vortex generators and other methods can indeed suppress low-frequency buffeting by destroying the ordinality of large-scale vortices (Evert et al., 2004). However, it generates high-frequency noise, which is unacceptable in aeroacoustics automotive wind tunnels. This passive method also has an effect on the axial static pressure gradient (Wickern et al., 2000). Some wind tunnels adopt active control methods to control low-frequency buffeting, such as Audi's ARC (Active Resonance Control) system, but the active maintenance means have higher maintenance costs in the later period, which limits the application of this control method in wind tunnels (Evert et al., 2004). The principle based on the active control method is also the destructive vorticity ordering. These control methods all require a large number of tests to determine the parameter settings and reflect on the side the lack of theoretical basis of the formation of buffeting phenomena. Based on the shortcomings and deficiencies of previous studies, this paper will study the relationship between the internal flow structure and the change of the pressure field based on a 1:15 3/4 open model wind tunnel. The result will provide theoretical basis for reducing buffeting phenomena.

\section{Methodology}

\subsection{Numerical Simulation}

The model selected for the simulation is a 1:15 3/4 open-jet return type scaled wind tunnel referring to the full-scale $3 / 4$ open jet automotive aerodynamics and aeroacoustics wind tunnel built in Shanghai Automotive Wind Tunnel Center (Yang, 2007), shown in Fig. 1, including the contraction section, test section, diffusion section, and extension section.. The size of the test section is $1517 \mathrm{~mm}$ (length) $\times 1185 \mathrm{~mm}$ (width) $\times 818 \mathrm{~mm}$ (height), and the nozzle size is $433 \mathrm{~mm}$ (width) $\times 283 \mathrm{~mm}$ (high). And it is the same size as the 1:15 scaled wind tunnel used as the experimental platform. The streamwise turbulence intensity is $0.6 \%$. The hydraulic diameter based on nozzle size $\mathrm{D}_{h}$ is 342 $\mathrm{mm}$. In order to simulate the pressure balance port, two pressure reference points were set at the two pressure balance ports, and the reference pressure was atmospheric pressure. The boundary condition applied at the outlet of the diffuser is set as FlowSplit Outlet. 
The angle of the collector was set to 0 degree. The breath opening was sealed to maximum the buffeting phenomenon. The velocity in the center of the nozzle $U_{e}$ was $25 \mathrm{~m} / \mathrm{s}$, due to the pressure fluctuations and velocity fluctuations could be most obvious at this condition (Jia, et al., 2001; Zheng, et al., 2006). No model was placed in the test section. According to the hydraulic diameter $\mathrm{D}_{\mathrm{h}}$ of the nozzle, the Reynolds number $\operatorname{Re}=5.7 \times 10^{5}$.

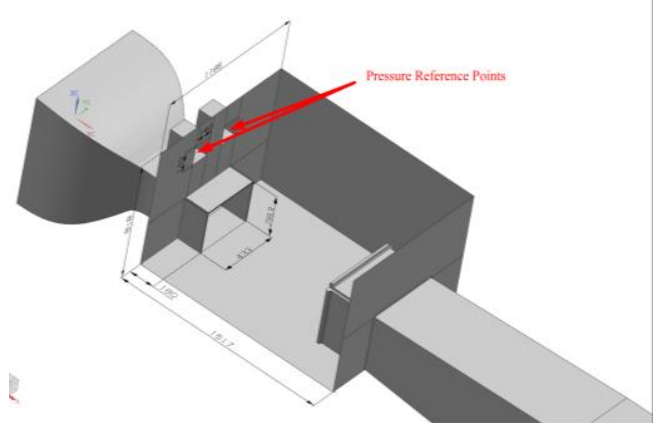

Fig. 1. Geometric model and pressure reference points.

The unsteady turbulent model used for the simulation was Large Eddy Simulation (LES) (Li et al., 2017). This paper's numerical simulation was performed with a commercial CFD package STAR$\mathrm{CCM}+$ (12.02). This model uses a discrete pressure solver based on the finite volume method. The boundary centered difference scheme was used for the diffusion terms and the second order upwind discrete scheme was used for the convection terms.

In the test section, the sampling area between the nozzle and the collector is shown in Fig. 2. The sampling area is used for data processing and POD analysis. The dimensionless dimension of the sampling area is $\mathrm{X} / \mathrm{D}_{\mathrm{h}}=2.5, \quad \mathrm{Y} / \mathrm{D}_{\mathrm{h}}=2.3$, and $\mathrm{Z} / \mathrm{D}_{\mathrm{h}}=1.5$. The sampling points were grid nodes. The initial flow field calculated by large eddy simulation was based on the results of the steady result. Because the large eddy simulation requires a certain settling time (Ma, et al., 2018a), the sampling of the numerical simulation was beginning at about $0.5 \mathrm{~s}$. Through frequency spectrum analysis of the sampling point between experiment and simulation, the starting time of the data used in the post-processing was judged. The final start time is $0.6 \mathrm{~s}$, the sampling frequency is $5000 \mathrm{~Hz}$, and the total sampling time is $3 \mathrm{~s}$.

The wind velocity selected for numerical simulation was $25 \mathrm{~m} / \mathrm{s}, \mathrm{Re}=5.7 \times 10^{5}$. The computational mesh used in this simulation was trimmer. This type of mesh generate boundary layer mesh and hexahedral mesh on the wall surface and in the core area, respectively. The mesh at the interface between the boundary layer and the core area is obtained by cutting a hexahedron. It is guaranteed that $\mathrm{y}^{+}<1, \mathrm{x}^{+}$ and $\mathrm{z}^{+}<50$, and the time step obtained based on the Courant number $\mathrm{CFL}<1$ is $5 \times 10^{-5} \mathrm{~s}$. The volume mesh and magnified area for grid near the surface is depicted in Fig. 3.

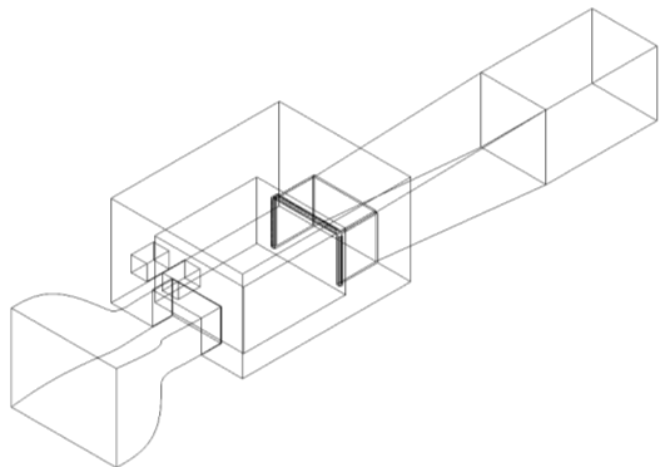

Fig. 1. Sampling area of numerical simulation.

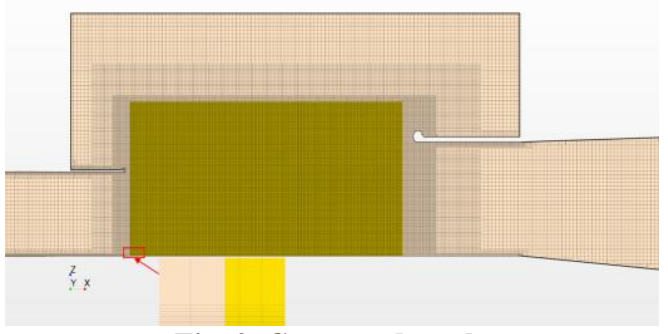

Fig. 2. Generated mesh.

\subsection{Experiment Validation}

In this research PIV is used for experimental validation. The layout of the PIV system during measurement is shown in Fig. 4. The pulse energy of the laser (Model Vlite-500 Pulse Laser, BEAMTECH) is $200 \mathrm{~mJ}$ and the wave length of laser pulse is $532 \mathrm{~nm}$. The CCD camera CFD(model TTS for PowerviewPuls) has a photo resolution of $6,600 \times 4,400$ pixels. The tracer particles used in the experiment were corn oil, and the corn oil entered the wind tunnel through the smoke generator. The diameter of the oil droplets was in the range of 1 to $5 \mu \mathrm{m}$. The sampling frequency used in this PIV test was $1.5 \mathrm{~Hz}$.

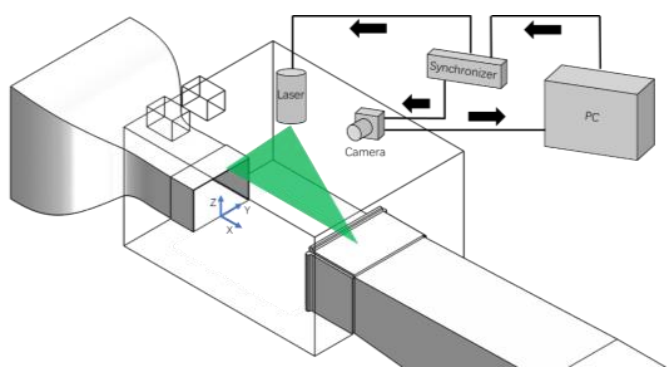

Fig. 3. PIV system layout and coordinate system.

Due to the limitations of the test site area, the focal distance of the PIV camera, and the laser energy, the jet shear layer from the nozzle to the collector was divided into three areas for measurement. As shown in Fig. 5, these measurement areas are named FOV1 3 (Field of View). The measurement plane was located in the symmetry plane $\mathrm{Y} / \mathrm{D}_{\mathrm{h}}=0$ in the wind tunnel, and the origin of the coordinates is set as shown in figure. In order to ensure the 
comprehensive measurement, the three measurement areas were overlaps. The principle of the size of the measurement area is that the average velocity field contains 0.05 to 0.95 times the incoming velocity, that is, the defined range of the jet shear layer. The laser and camera were recalibrated when the areas were changed, and it was confirmed that the different areas were all in the middle symmetry plane $\mathrm{Y} / \mathrm{D}_{\mathrm{h}}=0$.

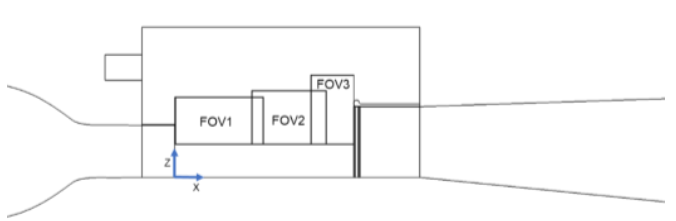

Fig. 4. PIV measurement areas.

\section{RESULTS}

\subsection{Compare Experiment with Simulation}

Select region FOV1 to validate the results obtained by numerical simulation. POD results are shown in Fig. 6.

It can be seen in Fig. 6 that the mode shapes at the initial section of the jet shear layer calculated by the LES are very similar to results obtained through experiment. These results are all represent the vortex shedding phenomenon of the KelvinHelmholtz $(\mathrm{K}-\mathrm{H})$ instability in the initial stage of the jet shear layer (Miles, 1959). The difference between PIV and LES results can be attributed to: different sampling frequencies, experimental interference, and different number of sampling points. The results obtained from the test were disturbed by the surrounding environment (fan instability, pressure balance flow and et. al), especially the edge of mode was disturbed more seriously. Numerical simulation can solve this problem effectively. The feasibility and reliability of LES method in this study can be verified, by comparing PIV with LES results.

\subsection{Unsteady Flow Behavbior}

The most obvious phenomenon of buffeting is the pressure fluctuation in the flow field of wind tunnel. This phenomenon can be intuitively shown through the pressure contour. Figure 7 shows the time sequence of instantaneous pressure distribution at the symmetry plane of the test section from nozzle to collector. This time sequence has a cycle of $\mathrm{T}=$ $0.05 \mathrm{~s}$ (frequency $\mathrm{f}=20 \mathrm{~Hz}$ ). The flow is from left to right. Defining the first graph of time series $t=0$ $\mathrm{s}$, along the time scale, the pressure in the test section has a significant reduction and recovery. When $\mathrm{t}=0 \mathrm{~s}$, there is a region of negative pressure in the jet shear layer induced by the movement of the vortex structures. These vortex structures change in size and shape. To demonstrate clearly, three distinct negative pressure regions are named as zone one, zone two and zone three along the flow direction. In the upstream of the jet shear layer, the negative pressure zone one and zone two caused by the vortex shedding are smaller than the downstream zone three, and the morphology of zone one and zone two remain relatively stable on the time scale. The right negative pressure zone three is larger in size and changes in time scale are also large. When $\mathrm{t}=0.01 \mathrm{~s}$, the negative pressure zone one and zone two generated by the vortex shedding from the jet shear layer have a very significant fusion phenomenon, which is very similar to the vortex pairing described in previous work (Yule, 1978; Liepmann et al., 1992; Grinstein, 2001; Ghasemi et al., 2016). This large negative pressure zone can be named as zone one plus two. At the same time, the pressure inside the plenum gradually decreases with the right negative pressure zone approaching the collector. When $t=0.02 \mathrm{~s}$, the pressure in the plenum has reduced to the minimum. At the same time, the right negative pressure zone three gradually breaks, and the edge becomes no longer smooth. The size of negative pressure zone one plus two is similar to the negative pressure zone three when $t=0 \mathrm{~s}$, which is formed on by the fusion with surrounding small negative pressure zones. When $\mathrm{t}=0.03$ and $0.04 \mathrm{~s}$, the downstream negative pressure zone in the jet shear layer rapidly break up and disappear near the collector. The pressure in the plenum rapidly rises. When $t=0.05 \mathrm{~s}$, the pressure distribution condition is similar to the original state at $\mathrm{t}=0 \mathrm{~s}$, that means a new circle period starts.

To demonstrate the change of the pressure field over time, the variation of the vorticity over time can be another effective parameter. The spanwise vorticity contour at the symmetry plane is shown in Fig. 8, corresponding to the pressure contour at each moment depicted in Fig. 7. According to the relation of pressure and vorticity (Rashidi et al., 2009; Ma, et al., 2018b), the negative pressure zones one and two can be considered as the vortex rings at the initial section of the jet shear layer. When $\mathrm{t}=0.01 \mathrm{~s}$, the vortex ring one and the vortex ring two get paired. The area corresponding to the negative pressure zone three is composed of some small vortices. These small vortex structures gather together and have similar mode of motion. This paper raises an assumption that the negative pressure zone three may be a vortex structure with larger size. This regularity will be mentioned in the following POD analysis for the velocity field. After the negative pressure zone three approaches the collector, the originally gathered vortices break up and eventually disappear. At the same time, the negative pressure zone one plus two gradually expands in size when moving downstream. Meanwhile, the number of internal small vortices increases obviously. In the next cycle period, the vortex one plus two and the vortex three will retain the same properties.

As analyzed above, the dimension of negative pressure zone increased gradually along with the flow, due to the pairing process of the vortex rings. The pressure in the plenum reduces, because the negative pressure zones break up and release the internal pressure, when these zones approaching the collector. Meanwhile, the pressure waves propagate 

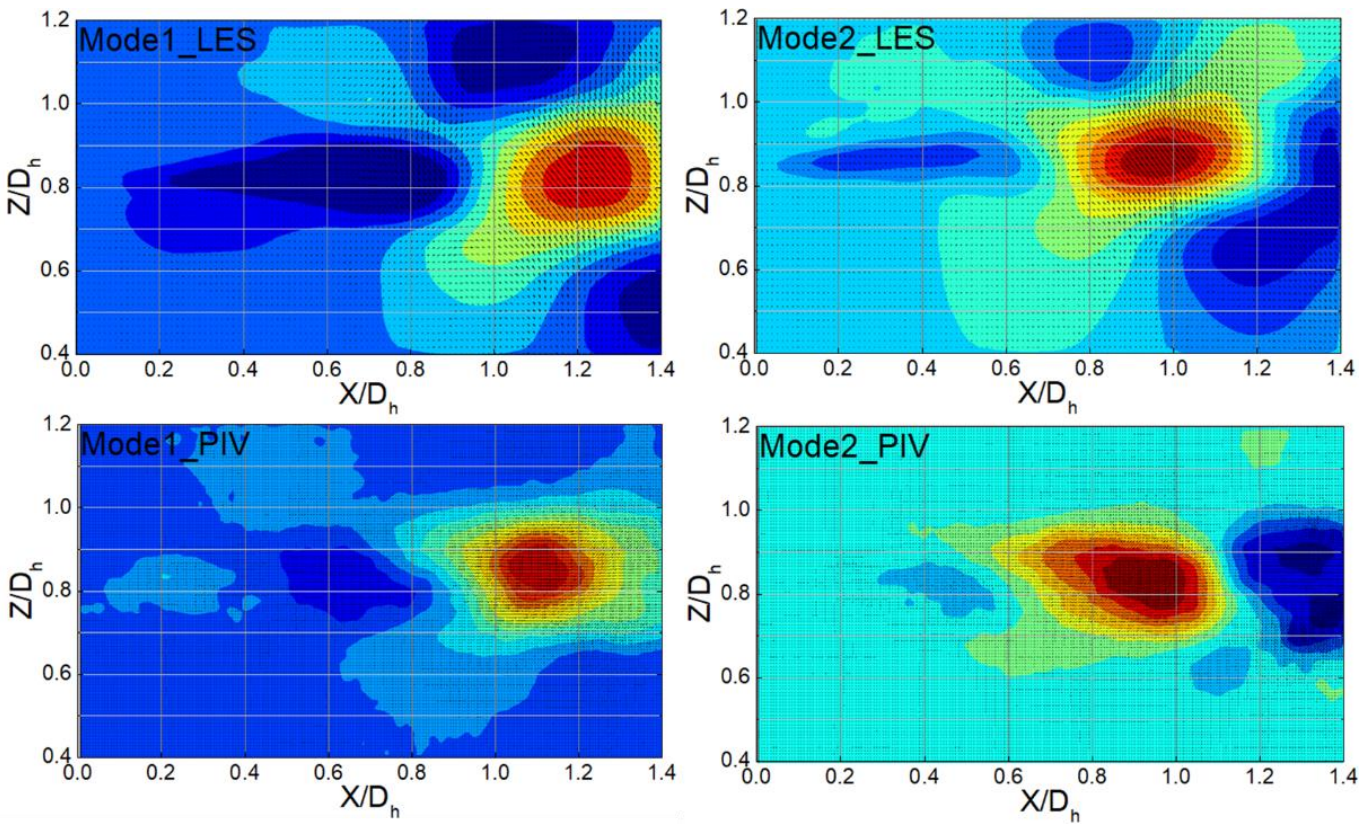

Fig. 6. Comparison of POD Results of PIV and LES (mode 1 and 2).

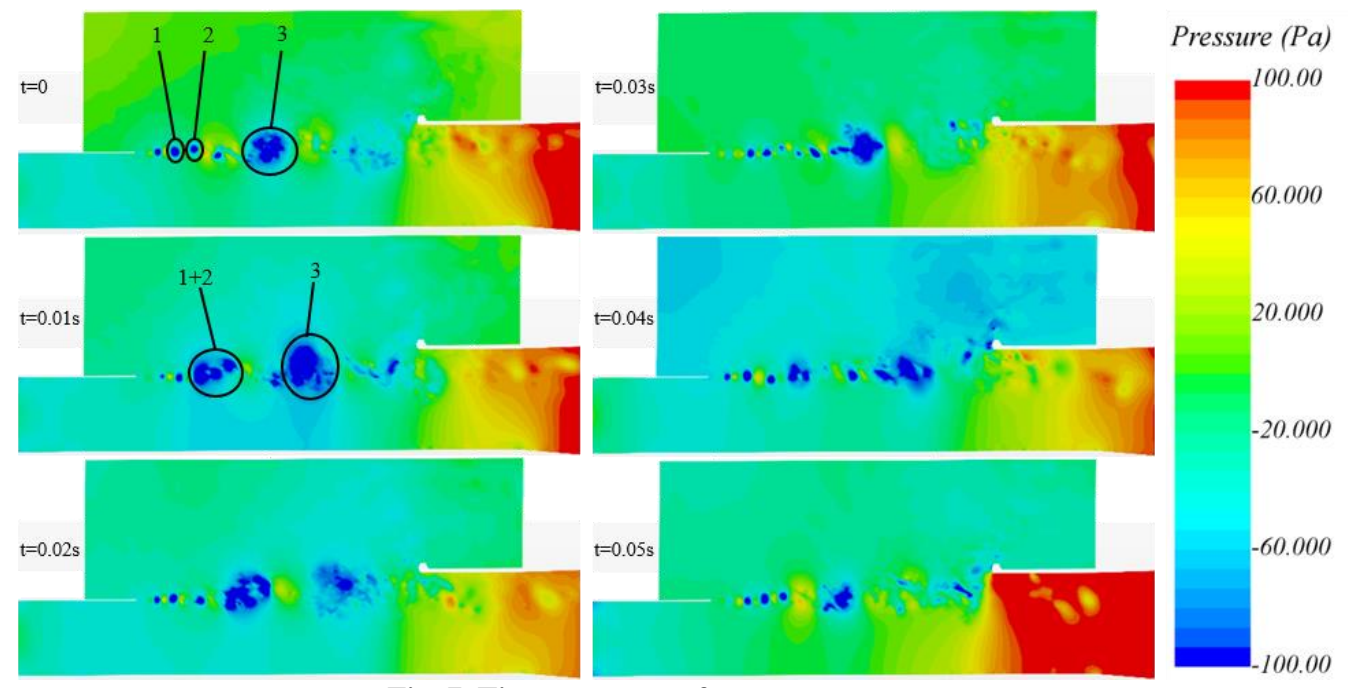

Fig. 7. Time sequence of pressure contours.

upstream at the speed of sound and excite the generation of shedding of upstream vortex rings. Since the vortex rings are paired once per pressure variation cycle period, the frequency of vortex-ring pairing and fusion could be the same as or similar to the frequency of pressure variation.

\subsection{D POD Analysis}

\subsubsection{Decomposition Based on Pressure}

In this section, POD analysis will be used to decompose and reconstruct one-dimensional pressure field in the mid-symmetry plane $\left(\mathrm{Y} / \mathrm{D}_{\mathrm{h}}=0\right)$ of the sampling area (Adrian et al., 2000). The dimensionless length of the middle symmetry plane is: $\mathrm{X} / \mathrm{D}_{\mathrm{h}}=2.5$ and the height is: $\mathrm{Z} / \mathrm{D}_{\mathrm{h}}=1.5$. This sampling area covers the area from the nozzle to the collector. The POD analysis is based on a pressure matrix composed of 1000 snapshots with a sampling frequency of $1000 \mathrm{~Hz}$, and the corresponding total sampling time is $1 \mathrm{~s}$.

The first 20 mode energy distribution of the onedimensional pressure field is shown in Fig. 9. The ratio of the first ten mode energy is about $84 \%$. The reason for this high energy ratio could be the regular pressure pulsation in the plenum chamber. This pulsation is widely acknowledged as lowfrequency buffeting phenomenon. Meanwhile, the Fig. 9 shows that the energy ratio of the first mode is about $42 \%$.

Using the first ten modes and the mean pressure field to reconstruct the pressure field can capture information with higher energy content in the pressure field. A time sequence within $0.05 \mathrm{~s}$ after reconstruction is shown in Fig. 10. Figure 10 shows a similar phenomenon as depicted in Fig. 7. 

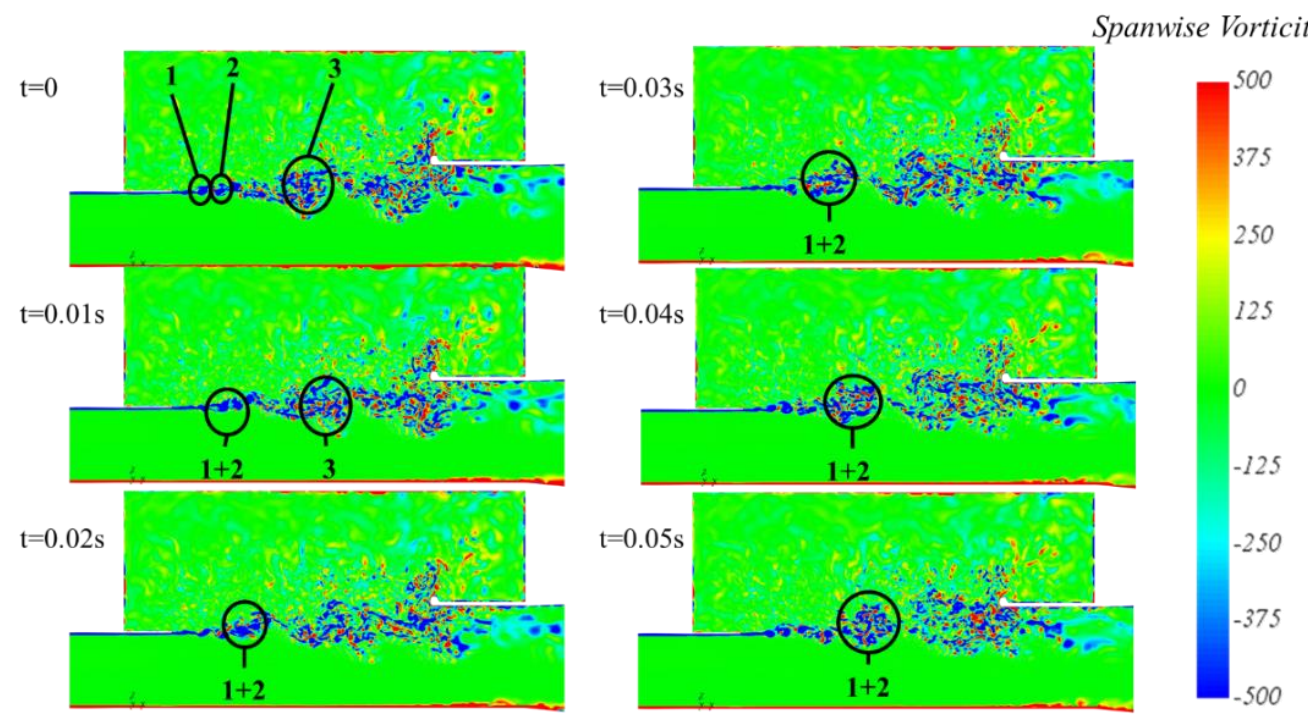

Fig. 8. Time sequence of vorticity contours.

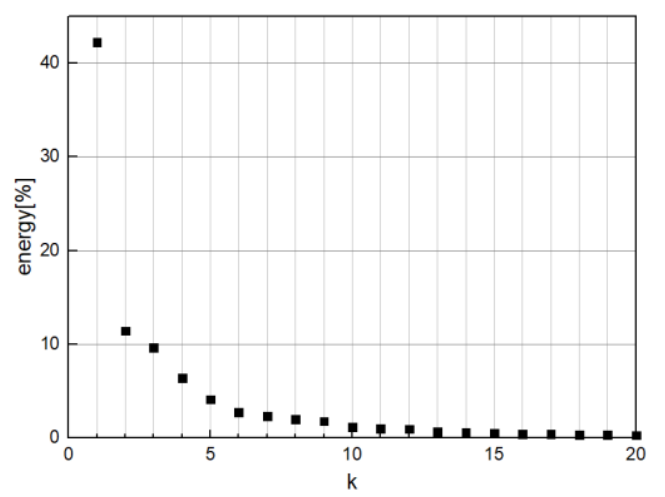

Fig. 9. Energy distribution of POD modes of pressure.

By performing FFT transform on the time coefficient matrix (mode coefficient matrix) of the first ten modes, important spectral information can be extracted. The spectrum of the mode time coefficient of the pressure field mode 1-10 is shown in Fig. 11. It can be found that the spectrum peak of the mode time coefficient of the pressure mode gather around $20 \mathrm{~Hz}$, which is also the dominant frequency of the low-frequency buffeting at this speed (Zheng, et al., 2007).

\subsubsection{Decomposition Based on Velocity}

A sa In this section, a two-dimensional POD analysis will be used to decompose the twodimensional velocity field of the symmetry plane $\left(\mathrm{Y} / \mathrm{D}_{\mathrm{h}}=0\right)$ in the sampling region. The dimensionless length of the middle symmetry plane is: $\mathrm{X} / \mathrm{D}_{\mathrm{h}}=2.5$, and the height is: $\mathrm{Z} / \mathrm{D}_{\mathrm{h}}=1.5$, covering the area from the nozzle to the collector. The two-dimensional POD analysis is based on a velocity matrix consisting of 1000 snapshots with a sampling frequency of $1000 \mathrm{~Hz}$, corresponding to a total sampling time of $1 \mathrm{~s}$.

The energy distribution of the first 20 modes of the 2D velocity field (1000 snapshot analysis results) and the cumulative energy distribution of different numbers of snapshots are shown in Fig. 12(a), where $\mathrm{k}$ is the mode number. Mode 1 and 2 of velocity account for $8.5 \%$ and $8.2 \%$ of the total kinetic energy. It is the highest energy in the jet shear layer, that means these two can be considered as the dominant mode. For different number of snapshots, the cumulative energy distribution approaches $100 \%$ from 250, 500, 750, and 1000 frames, as shown on the right in Fig. 12(b). And the difference between the curves of 750 frames and 500 frames is small. Figure 13 shows the slopes of cumulative relative energy of different number of snaps.

It is obvious that slopes of 250,500 and 750 snaps all skip to zero abruptly. So, 1000 snaps for reconstruction could be convergent and reliable.

For POD analysis of a two-dimensional velocity field in the mid-symmetry plane $\left(\mathrm{Y} / \mathrm{D}_{\mathrm{h}}=0\right)$, approximately $50 \%$ of the kinetic energy is captured by the first 20 modes. The first ten modes are shown in Fig. 14 and Fig. 15. The energy of each mode is marked in the upper left corner. The mode of streamwise velocity is on the left and the mode of vertical velocity is on the right. Only a single mode in the pairs is given in brief. The first ten POD mode of velocity account for $40 \%$ of the kinetic energy. The pairs with the phase difference $\pi / 2$ (modes 1 and 2, 3 and 4,6 and 7,9 and 10) describe the waves with downward trend (showed in Fig. 14), while single modes (modes 5 and 8) describe the waves without downward trend (showed in Fig. 15).

Based on the FFT spectrum of the mode time coefficient (mode coefficient) (Fig. 16), mode 1 and 2 have the same peak frequency of $21 \mathrm{~Hz}$. This frequency is also the dominant frequency of low frequency buffeting at this wind speed. The mode pair 3 and 4, 6 and 7 also have the same peak frequency of $21 \mathrm{~Hz}$, but the spectrums have different second peaks. These second peaks may 

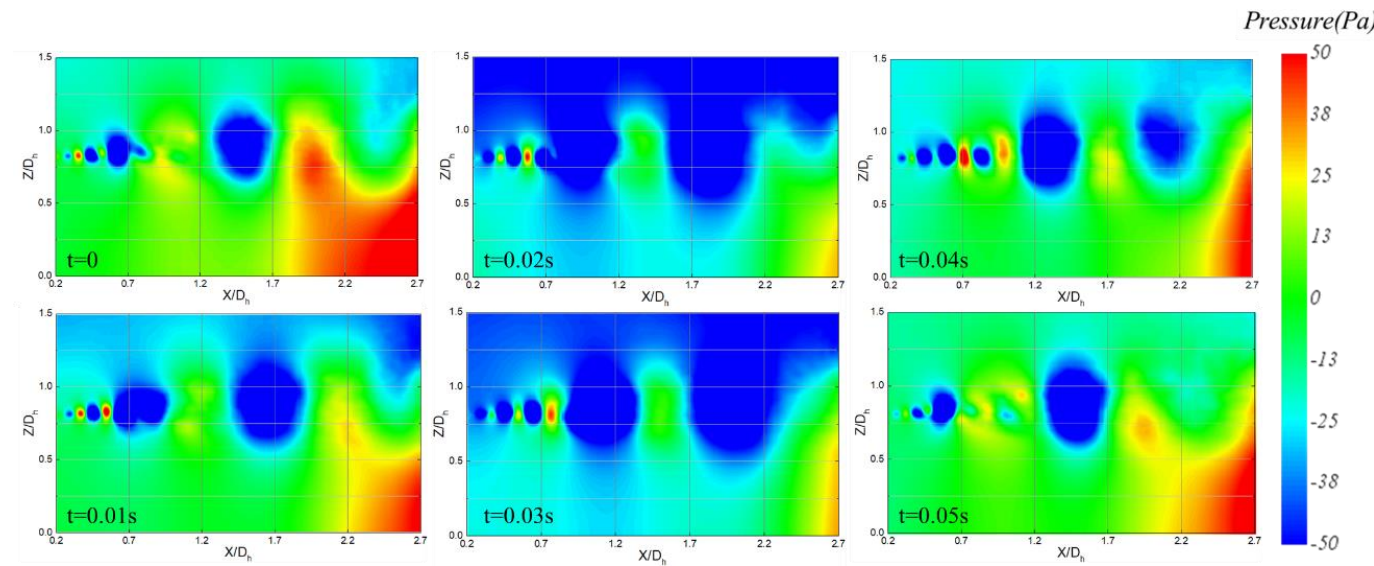

Fig. 10. Time sequence of reconstructed pressure field.

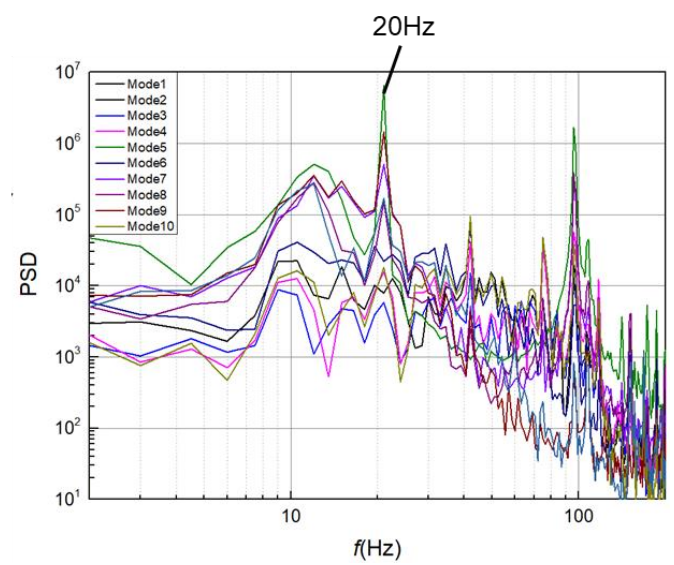

Fig. 11. Time coefficient spectrum of mode1-10.

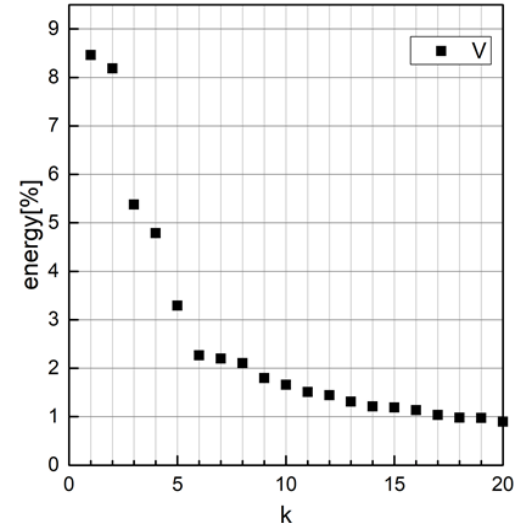

Fig. 12. (a). Energy distribution of the first 20 modes of velocity.

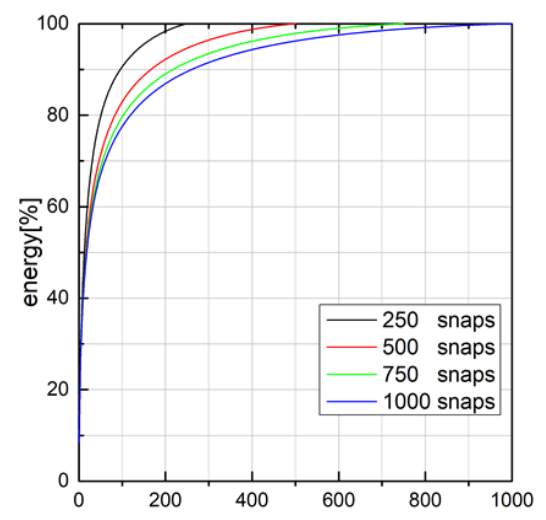

Fig. 12. (b). Accumulative energy distribution.

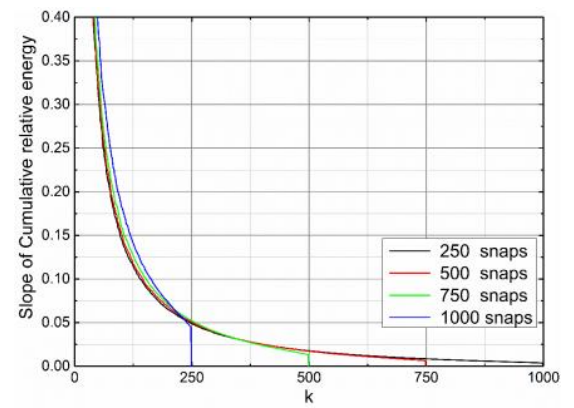

Fig. 13. Slope of cumulative relative energy. 

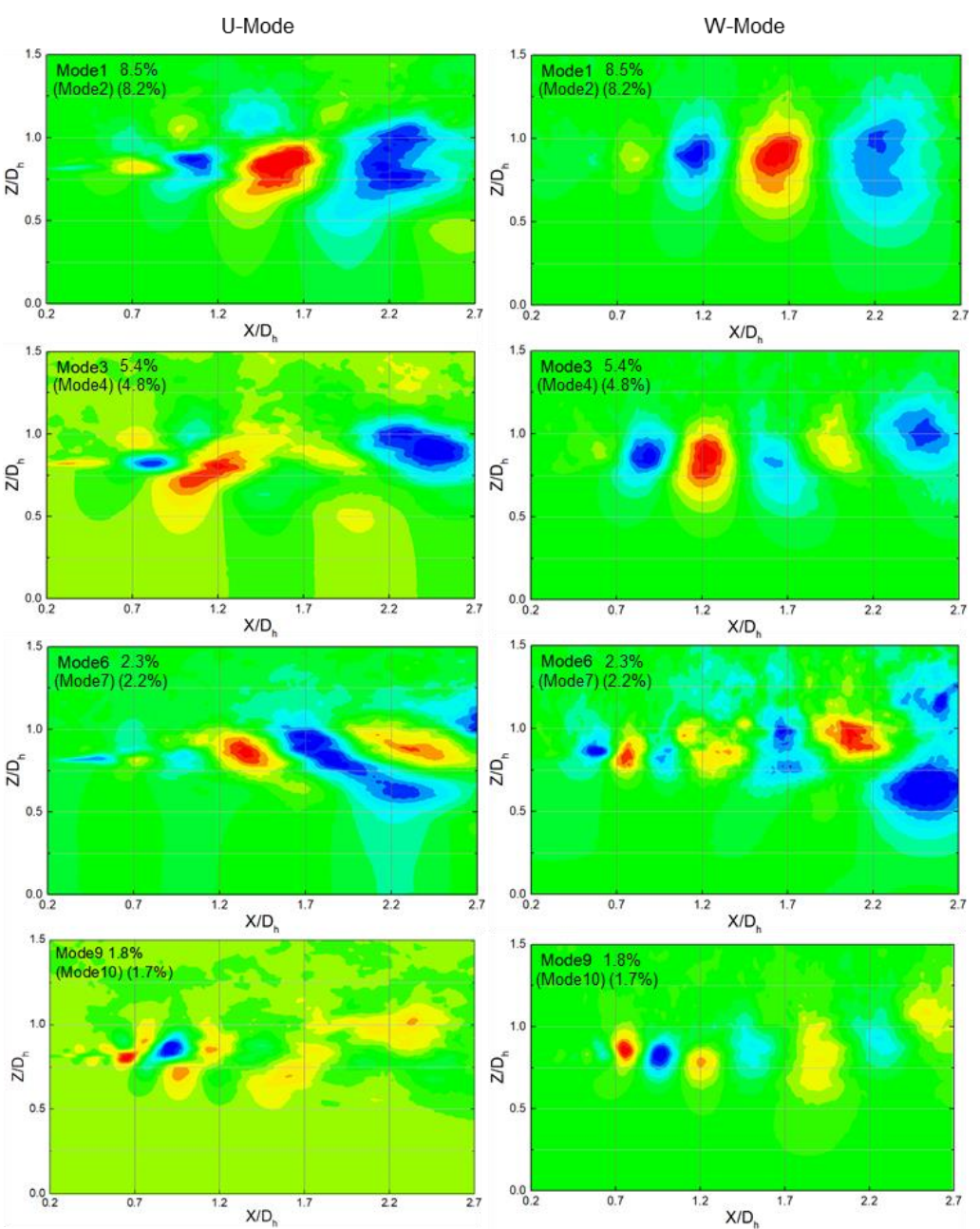

Fig. 14. Modes of velocity describing traveling waves.
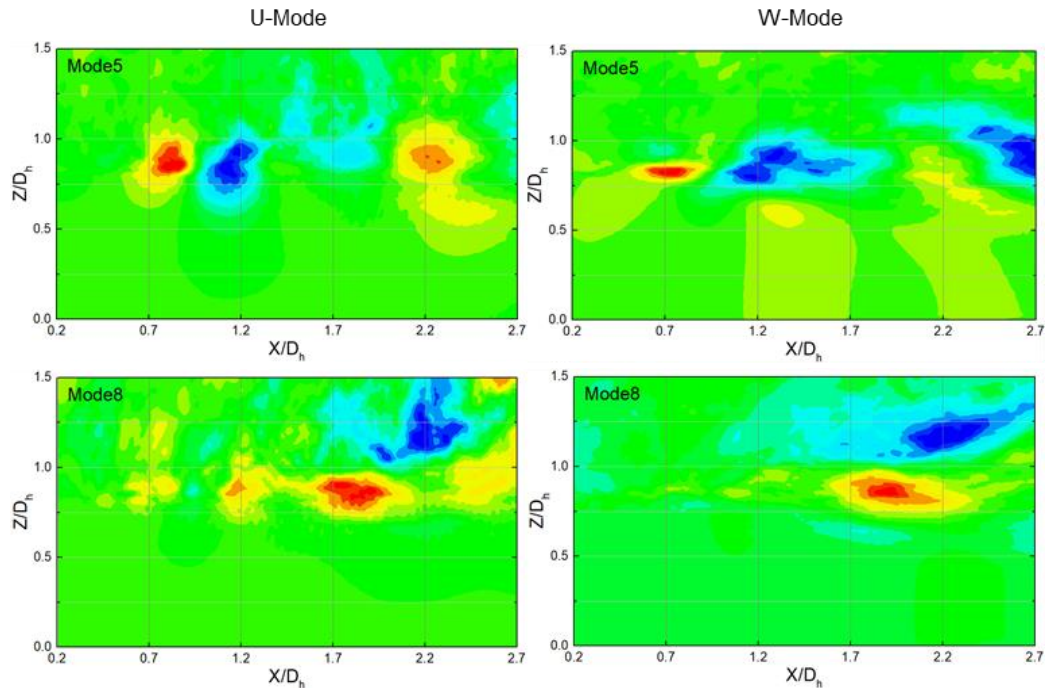

Fig. 15. Modes of velocity describing non-traveling waves.

correspond to other vortex structures and mode pair 9 and 10 are located in the vortex shedding area, and have a peak frequency of $42 \mathrm{~Hz}$. This peak frequency is twice over the mode 1 and 2 peak frequency. Therefore, they are corresponding to the vortex rings generated after shedding

It is difficult to determine what kind of flow structure mode 5 representing, but it has a high degree of similarity to mode 3 . 


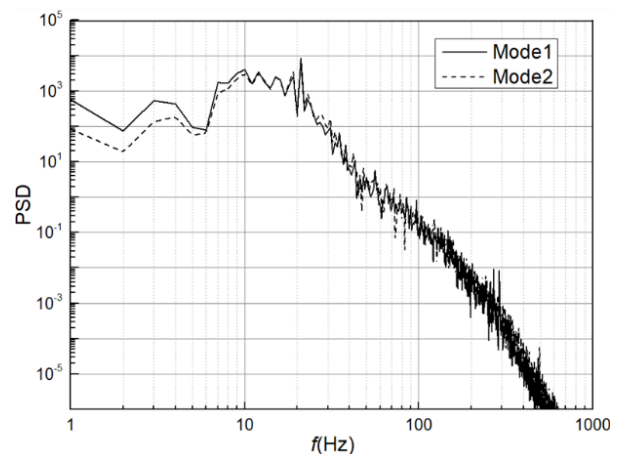

Fig. 16. (a). Spectrum of time coefficients of mode 1 and 2.
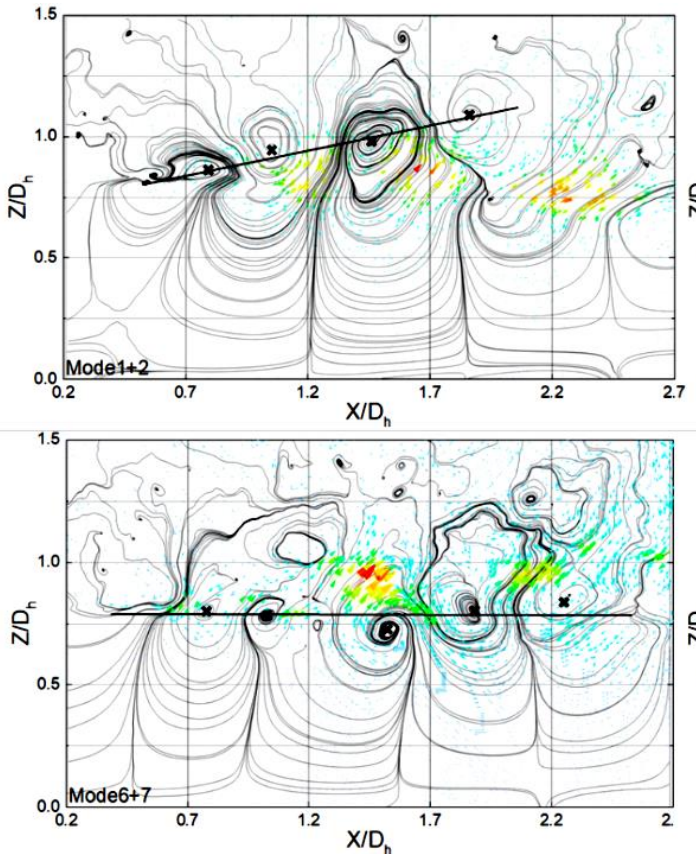

Fig. 17. Reconstructed streamlines of velocity modes.

The flow characteristics represented by modes can be analyzed with the method of using mode matrix and mode time coefficient matrix to reconstruct different modes. The streamline patterns are selected as the evaluation index of the vortices. This vector fields do not contain the mean field. The streamline patterns at a certain moment is shown in Fig. 17. The center of the vortices reconstructed by different modes is marked. In this figure, the position of the vortex is clearly visible. Mode 1 and Mode 2 describe the vortex ring moving downstream, which is located between the regions of vortex ring pairing and the rupture of the lowpressure zone. The vortex cores is at an angle of about 10 degree with the $X$ axis. The center of the vortex core reconstructed by mode 3 and 4 is at an angle of approximately -10 degree with the $\mathrm{X}$-axis. The vortex cores reconstructed by mode 6 and 7 is almost parallel to the $\mathrm{X}$ axis. The vortex cores reconstructed by mode 9 and 10 is similar to the center of the vortex reconstructed by mode 3 and 4, which is approximately -10 degree.
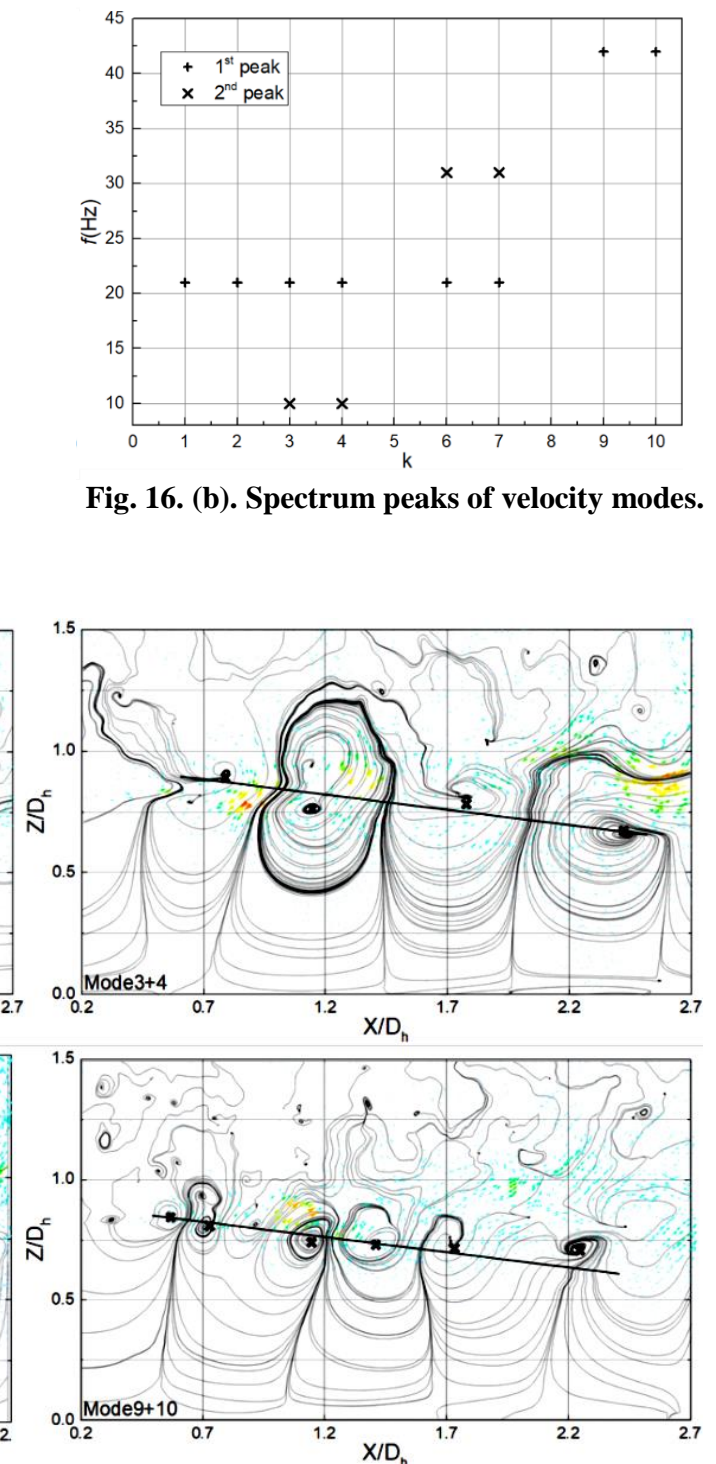

Fig. 16. (b). Spectrum peaks of velocity modes.

The linear combinations of these vortex structures travel downstream and knock on the collector. The pressure waves generated from this motion reflow to the nozzle region and excite the upstream vortex structures to be more coherent. Reconstructing vortex structures by different modes has different propagation directions. Therefore, it is necessary to compare these modes with the position of the collector. As shown in Fig. 18, the relationship between the vortex propagation direction and the position of the collector is different for different mode pairs. The propagation direction of the mode vortices for the 1 st and 2 nd reconstructed flow vortices is closer to the collector than the other three pairs.

As mentioned above, the dominant mode characteristics of the velocity in the jet shear layer have the same characteristic frequency as the dominant low frequency buffeting frequency at this velocity $(25 \mathrm{~m} / \mathrm{s})$. Mode 1 and 2 with the highest energy content has a characteristic frequency of 21 Hz. The first 3 pairs (1 and 2, 3 and 4, 6 and 7) have 


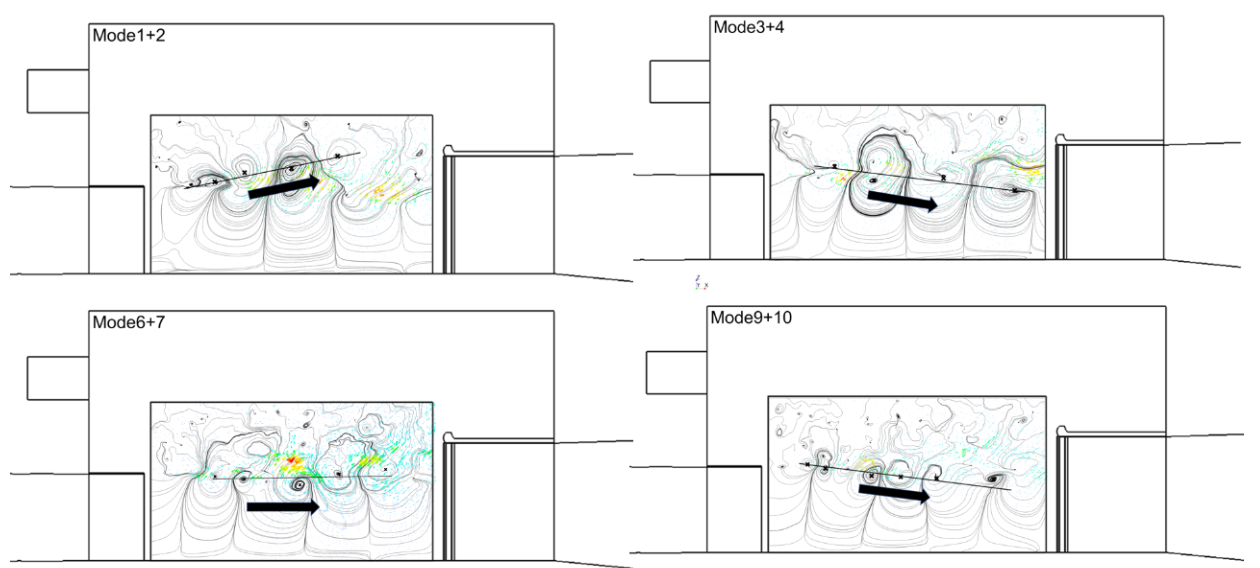

Fig. 18. The relative position between the vortices movement trend and the collector.

the same main peak frequency and different subpeak frequencies. Thus, represents the vortex rings and other frequency vortices generated after pairing and fusion.

\section{CONCLUSION}

In this paper, numerical simulation (LES) was used to study the jet shear layer of a $1: 15$ scaled $3 / 4$ open-jet wind tunnel, and the temporal and spatial evolution of the vortex structure in the jet shear layer was revealed. The main results are as follows:

1. The results show that the initial section of the jet shear layer is dominated by regular vortex rings, and the vortex ring pairing phenomenon occurs near $\mathrm{X} / \mathrm{D}_{\mathrm{h}}=1$.

2. The pressure contours of the symmetry plane shows that there is a certain regularity in the change of the pressure in the plenum, and it is related to the negative pressure region induced by the vortex movement.

3. By performing POD decomposition on the twodimensional velocity field of the symmetry plane, mode pairs with high kinetic energy represent the vortex rings formed after vortex pairing. These mode pairs have the same characteristic frequency as the dominant frequency of low-frequency buffeting.

\section{ACKNOWLEDGEMENTS}

Thanks for project 11502171 financially supported by National Natural Science Foundation of China.

\section{REFERENCES}

Adrian, R. J., K. T. Christensen and Z. C. Liu (2000). Analysis and interpretation of instantaneous turbulent velocity fields. Experiments in Fluids 29, 275-290.

Amandolese, X. and C. Vartanian (2010). Reduction of $3 / 4$ open jet low-frequency fluctuations in the S2A wind tunnel. Journal of Wind Engineering \& Industrial
Aerodynamics 98.10, 568-57.

Arnette, A. Stephen, T. D. Buchanan and M. Zabat (1999). On low-frequency pressure pulsations and static pressure distribution in open jet automotive wind tunnels. SAE Technical Paper.

Evert, F., and H. Miehling (2004). Active suppression of buffeting at the Audi AAWT: Operational Experiences and Enhancements of the Control Scheme. SAE Technical Paper.

Ghasemi, A., V. Roussinova, R. M. Barron and R. Balachandar (2016). Large eddy simulation of the near-field vortex dynamics in starting square jet transitioning into steady state. Physics of Fluids 28(8).

Grinstein, F. F. (2001). Vortex dynamics and entrainment in rectangular free jets. Journal of Fluid Mechanics 437, 69-101.

Jia, Q., Z. Yang and Q. Li (2001). Test research of the flow field inside the test section of the automotive wind tunnel. Journal of Experiments in the Fluid Mechanics 6,007.

Li, Q., L. Zhong, Y. Wang, Z. Yang and M. M. Rashidi(2017). Multi-parameter optimization of automotive rear view mirror region for reducing aerodynamic noise. Noise Control Engineering Journal 66(1), $11-26$.

Liepmann, D. and M. Gharib (1992). The role of streamwise vorticity in the near-field entrainment of round jets. Journal of Fluid Mechanics 245, 643-668.

Ma, Y., R. Mohebbi, M. M. Rashidi and Z. Yang (2018a). Numerical simulation of flow over a square cylinder with upstream and downstream circular bar using lattice Boltzmann method. International Journal of Modern Physics C.

Ma. Y., R. Mohebbi, M. M. Rashidi and Z. Yang (2018b). Study of nanofluid forced convection heat transfer in a bent channel 
Q. Jia et al. / JAFM, Vol. 12, No. 5, pp. 1359-1369, 2019.

by means of lattice Boltzmann method. Physics of Fluids 30,3

Manuel, G. S., J. K. Molloy and P.S. Barna (1992). Effect of collector configuration on test section turbulence levels in an open-jet wind tunnel. NASA Langley Technical Report Sever.

Michel, U. and E. Froebel (1985). Turbulence at far downstream positions in the open working section of the German-Dutch Wind Tunnel DNW. DFVLR IB: 22214-85.

Miles, J. (1959). On the generation of surface waves by shear flows Parts 3.KelvinHelmholtz instability. Journal of Fluid Mechanics 6(4), 583-598.

Morel, Thomas (1979). Experimental study of a jet-driven Helmholtz Oscillator. Journal of Fluids Engineering 101(3), 383-390.

Rashidi, M. M. and H. Shahmohamadi. (2009). Analytical solution of the three-dimensional Navier-Stokes equations for the flow near an infinite rotating disk. Communications in Nonlinear Science \& Numerical Simulation 14(7), 2999-3006.

Rennie, M. (2000). Effect of jet length on pressure fluctuations in 3/4-open jet wind tunnels. Motor Industry Research Association Vehicle Aerodynamics 2000 Symposium.

Rossiter, J. E. (1964). Wind tunnel experiments on the flow over rectangular cavities at subsonic and transonic speeds. Ministry of Aviation; Royal Aircraft Establishment; RAE Farnborough.

Wickern, G., W. V. Heesen and S. Wallmann (2000). Wind tunnel pulsations and their active suppression. SAE 2000 World Congress: e69-e70.

Yang, Z. (2007). Shanghai automotive wind tunnel center project. Proc. Stuttgart Symposium. Germany: Expert Verlag.

Yule, A. J. (1978). Large scale structures in the mixing layer of a round jet. Journal of Fluid Mechanics 89(3), 413-432.

Zheng, Z., and Wang, Y. (2007). An experimental study on the suppression of the low-frequency pulsation of the model wind tunnel. Automotive Engineering.

Zheng, Z., W. Peng and X. Jin (2006). The study of automotive wind tunnel acoustic control. Noise \& Vibration Control 3,020. 